\title{
Microwave-induced thermoacoustic tomography using multi-sector scanning
}

\author{
Minghua Xu, Geng Ku, and Lihong V. Wang ${ }^{\mathrm{a})}$ \\ Optical Imaging Laboratory, Biomedical Engineering Program, Texas A\&M University, 3120 TAMU, \\ College Station, Texas 77843-3120
}

(Received 26 March 2001; accepted for publication 18 June 2001)

\begin{abstract}
A study of microwave-induced thermoacoustic tomography of inhomogeneous tissues using multisector scanning is presented. A short-pulsed microwave beam is used to irradiate the tissue samples. The microwave absorption excites time-resolved acoustic waves by thermoelastic expansion. The amplitudes of the acoustic waves are strongly related to locally absorbed microwave-energy density. The acoustic waves may propagate in all spatial directions. A focused ultrasonic transducer is employed to acquire temporal acoustic signals from multiple directions. Each detected signal is converted into a one-dimensional (1D) image along the acoustic axis of the transducer. The crosssectional images of the tissue samples are calculated by combining all of the 1D images acquired in the same planes. (C) 2001 American Association of Physicists in Medicine.
\end{abstract}

[DOI: $10.1118 / 1.1395037]$

Key words: microwave, thermoacoustics, tomography, imaging, sector, scan

\section{INTRODUCTION}

Microwave-induced thermoacoustic tomography of biological tissues has recently attracted considerable interest. ${ }^{1-3}$ With this technique, a short-pulsed microwave beam is used to irradiate tissue samples. The tissue absorbs the microwave energy and excites thermoacoustic waves by thermoelastic expansion. The generated acoustic waves carry information about the microwave absorption properties of the sample. The different absorption properties among different types of tissue permit the construction of a distribution of microwave absorption in a homogeneous acoustic medium.

The microwave penetration depth in most soft tissues lies somewhere between that of the fat tissue, which lacks water and salt, and muscle tissue, which is abundant in water and salt. ${ }^{2}$ Specifically, the penetration depths for fat and muscle tissue at $3-\mathrm{GHz}$ microwave are $9 \mathrm{~cm}$ and $1.2 \mathrm{~cm}$, respectively. The wide range of microwave absorption coefficients among various other tissues can lead to a high imaging contrast for biological tissues. However, it is difficult to achieve good spatial resolution using pure microwave imaging of biological tissues because of the long wavelength of microwaves, e.g., $3 \mathrm{~cm}$ at $3 \mathrm{GHz} .{ }^{4,5}$ This problem can be overcome by the use of microwave-induced thermoacoustic waves. Because the velocity of acoustic waves in soft tissue is nearly $1.5 \mathrm{~mm} / \mu \mathrm{s}$, thermoacoustic signals at mega $\mathrm{Hz}$ can provide millimeter spatial resolution.

The intensities of the microwave-induced thermoacoustic signals are far lower than the ultrasonic pulses used in purely ultrasound imaging (ultrasonography). However, a unique advantage of thermoacoustic tomography is the detection of the inhomogeneous microwave absorption property of tissues when the acoustic property is homogeneous. Such a capability may lead to early detection of cancer.

Key problems in microwave-induced thermoacoustic tomography are the measurement of acoustic signals excited by microwave pulses and the construction of images from the acquired data. One approach is to use focused ultrasonic transducers to localize the thermoacoustic sources. The mature scanning techniques (linear and sector scans) in ultrasonography can be used to detect the thermoacoustic signals. ${ }^{6}$ Each scan line may reflect the profile of the medium along the acoustic axis of the focused transducer. However, an acoustic source has a strong direction of radiation, especially if the surface is relatively smooth, from which the acoustic energy is mainly transmitted out in one direction. The higher the frequency of the acoustic wave is, the stronger the radiation direction is. The thermoacoustic signals caused by microwave pulses are composed of highfrequency components as well as low-frequency components, and the intensities of the high-frequency components are far less than the ultrasonic pulses used in ultrasonography. Only if its acoustic axis is nearly perpendicular to the surface of the acoustic source, can the focused transducer acquire enough high-frequency components for accurate spatial localizations of the thermoacoustic sources. Therefore, it is necessary, with this method, to scan the sample from all possible directions by a focused transducer. Since the thermoacoustic wave is weak, in order to get a good signal-andnoise ratio (SNR), we use a focused transducer with a big aperture area in our initial study, because the SNR is proportional to the square root of the aperture area.

Here we present our study on microwave-induced thermoacoustic tomography of inhomogeneous tissues by a twodimensional (2D) full-directional scan-the combination of multiple-sector scans at various positions on a circle around the sample. Each detected time-resolved signal is converted into a one-dimensional image along the acoustic axis. The axial resolution is obtained by measuring the temporal profiles of the acoustic signals, and the lateral resolution is mainly determined by the focal diameter of the transducer. 
The two-dimensional (2D) cross-sectional images are calculated from the data acquired in the same planes. Some images of biological-tissue samples are achieved experimentally. These images clearly reveal the boundaries of different tissues as well as their locations, which are in good agreement with the actual samples.

\section{THEORY}

\section{A. Thermoacoustic wave}

When the microwave pulse duration is very short, the thermal conduction time is far greater than the thermoacoustic transit time. In practice, the duration of the microwave pulse is less than $1 \mu \mathrm{s}$, which meets this criterion. ${ }^{1}$ In this case, the heat diffusion's effect to the thermoacoustic wave in the tissue can be neglected. Consequently, the generation of thermoacoustic wave by deposition of microwave energy can be described by the following equation: ${ }^{7}$

$$
\left(\boldsymbol{\nabla}^{2}-\frac{1}{c^{2}} \frac{\partial^{2}}{\partial t^{2}}\right) p(\mathbf{r}, t)=-\frac{\beta}{C_{p}} \frac{\partial I(t)}{\partial t} A(\mathbf{r}),
$$

where $p(\mathbf{r}, t)$ is the thermoacoustic pressure at position $\mathbf{r}$ and time $t, c$ is the speed of sound, $\beta$ is the isobaric volume expansion coefficient, $C_{p}$ is the heat capacity, $I(t)$ is the temporal profile of the microwave pulse, and $A(\mathbf{r})$ is the fractional energy-absorption per unit volume of soft tissue at position $\mathbf{r}$, which is proportional to the microwave absorption coefficient of the tissue at position $\mathbf{r}$.

Equation (1) shows that the amplitudes of the thermoacoustic waves are strongly related to locally absorbed microwave-energy density, i.e., the local microwave absorption coefficient or penetration depth. Considering a spherically symmetric deposition of microwave energy with radius $R$,

$$
A(r)=A_{0} U(-r+R),
$$

where the step function

$$
U(\xi)= \begin{cases}1, & \xi \geqslant 0 \\ 0, & \xi<0\end{cases}
$$

Assuming a delta pulse of the form $I(t)=I_{0} \delta(t)$, the thermoacoustic pressure at detection position $r$ for $t>0$ is found from Eq. (1) to be ${ }^{7}$

$$
\begin{aligned}
p(r, t)= & \frac{\beta I_{0} c^{2}}{C_{p}} \frac{A_{0}}{2 r}(r-c t)[U(-r+R+c t) \\
& +U(r+R-c t)] .
\end{aligned}
$$

Moreover, the first derivative of the thermoacoustic pressure is written as

$$
\frac{\partial p(r, t)}{\partial t}=-\frac{\beta I_{0} c^{3}}{2 r C_{p}} A_{0} U(-|c t-r|+R) .
$$

Equation (4) indicates that the first derivative of the thermoacoustic pressure is a mapping of the spherically spatial energy deposition of Eq. (2). Therefore, we can use either the detected thermoacoustic pressure or its first derivative to

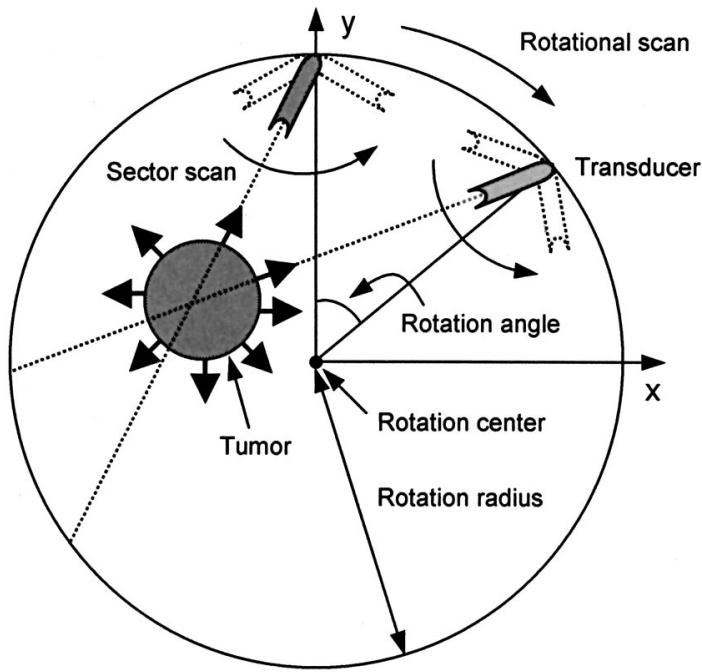

FIG. 1. A diagram of the multi-sector scan scheme.

construct the microwave absorption distribution and the latter is found to be better. In general, a large medium can be regarded as a set of small media or thermoacoustic sources, which have different microwave absorption coefficients. Through the following multi-sector scan method, each small thermoacoustic source can be localized.

\section{B. Multi-sector scan}

A diagram of the $2 \mathrm{D}$ multi-sector scanning scheme is shown in Fig. 1. A rectangular coordinate system $(x, y)$ is set up for reference. Each sector-scan frame has a set of scan lines, which originate from the same location and radiate out in different directions as in ultrasonography. The origins of different frames are set on a circle around the sample so that the focused transducer may detect the signals from all directions in the same plane. For convenience, the center and radius of the circle are referred to as the rotation center and the rotation radius, respectively.

The microwave absorption and sample heating occur in a very short time, and the propagation velocity of the electromagnetic wave is far greater than that of an ultrasonic one. Therefore, it is reasonable to assume that the sample expansion resulting from the microwave pulses causes acoustic waves instantaneously. The distance between the thermoacoustic source and the transducer is simply calculated by multiplying the time of arrival by the velocity of the acoustic wave. Therefore, at each scan line, the thermoacoustic pressure along the acoustic axis induced by the microwave pulses can be obtained directly from the detected temporal signals.

All scan lines are used to construct a cross-sectional image by the method of linear interpolation. A sector frame can only detect part of the sound source. Figure 2(a) shows the same $x-y$ plane as in Fig. 1. The transducer is now set at the position of point $O_{1}$. A local polar coordinate system $(d, \alpha)$ is set up for the sector frame with the origin $O_{1}$, and its polar axis is through the rotation center point $O$. The angle $\theta$ between the polar axis and $y$ axis is called the rotation angle. 


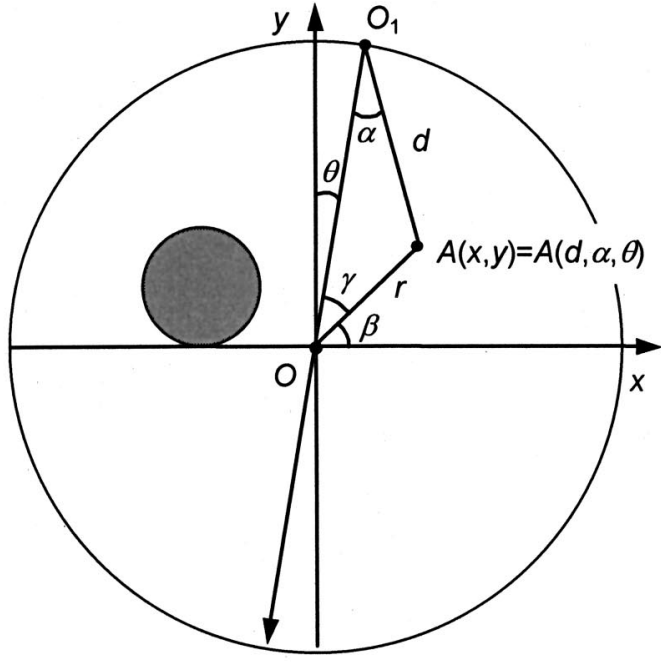

(a)

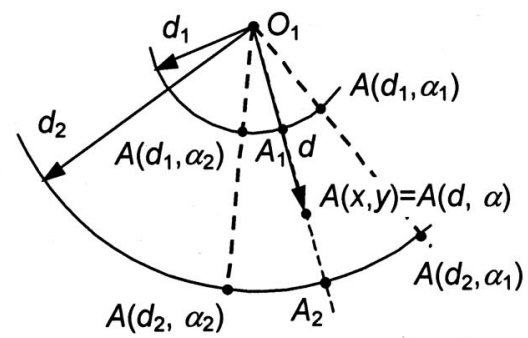

(b)

FIG. 2. (a) The rectangular coordinate system $(x, y)$ and the local polar coordinate system $(d, \alpha, \theta)$; (b) the diagram of the linear interpolation.

The rotation radius between point $O$ and $O_{1}$ is $R$. The distance between the thermoacoustic source and the transducer is $d$. Therefore, at each scan line, the point $A(x, y)$ in the rectangular coordinate system can be denoted as $A(d, \alpha, \theta)$ in the local polar coordinate system with rotation angle $\theta$. The relationship between $(x, y)$ and $(d, \alpha, \theta)$ is determined by the following equations:

$$
\begin{aligned}
& d=\sqrt{r^{2}+R^{2}-2 r R \cos \gamma}, \\
& \theta=\arcsin (r \sin \gamma / d), \\
& \beta=\arctan (y / x), \\
& \gamma=\pi / 2-\alpha-\beta, \\
& r=\sqrt{x^{2}+y^{2}} .
\end{aligned}
$$

In experiments, a series of discrete data are acquired in each local polar coordinate system. We use linear interpolations to project the data from each local polar coordinate system to the rectangular coordinate system. For simplicity, we neglect the symbol $\theta$ in the following description. As shown in Fig. 2(b), suppose that point $(d, \alpha)$ is among the measured data $A\left(d_{1}, \alpha_{1}\right), A\left(d_{2}, \alpha_{1}\right), A\left(d_{1}, \alpha_{2}\right)$, and $A\left(d_{2}, \alpha_{2}\right)$, i.e., $\alpha_{1}$ $\leqslant \alpha \leqslant \alpha_{2}, d_{1} \leqslant d \leqslant d_{2}$, where $d_{i}(i=1,2)$ is the distance be- tween the thermoacoustic source and the transducer and is calculated by multiplying the time of arrival $t_{i}(i=1,2)$ by the velocity of the acoustic wave $c$ in the medium, i.e., $d_{i}$ $=c t_{i}(i=1,2)$. Then $A(d, \alpha)$ can be calculated by

$$
\begin{aligned}
& A(d, \alpha)=\frac{d-d_{1}}{d_{2}-d_{1}}\left(A_{2}-A_{1}\right)+A_{1}, \\
& A_{1}=A\left(d_{1}, \alpha_{1}\right)+\frac{A\left(d_{1}, \alpha_{2}\right)-A\left(d_{1}, \alpha_{1}\right)}{\alpha_{2}-\alpha_{1}} \times\left(\alpha-\alpha_{1}\right), \\
& A_{2}=A\left(d_{2}, \alpha_{1}\right)+\frac{A\left(d_{2}, \alpha_{2}\right)-A\left(d_{2}, \alpha_{1}\right)}{\alpha_{2}-\alpha_{1}}\left(\alpha-\alpha_{1}\right) .
\end{aligned}
$$

Function $A$ could be the thermoacoustic pressure $p(t)$ or the derivative $d p(t) / d t$. The derivative is helpful for improving the sharpness of the boundaries between different tissues, which can be calculated through an inverse fast Fourier transformation (FFT) as

$$
\frac{d p(t)}{d t}=\mathrm{FFT}^{-1}\{i \omega p(\omega)\},
$$

where $p(\omega)$ is the Fourier transformation of $p(t)$.

In this way, we can compute each image $A_{\theta}(x, y)$ from each sector frame $A(d, \alpha)$ at rotation angle $\theta$. A full 2D cross-sectional image is obtained with the following summation:

$$
A(x, y)=\sum_{\theta} A_{\theta}(x, y) .
$$

\section{EXPERIMENTAL METHOD}

We use a focused transducer to implement a multi-sector scan. Figure 3 shows the experimental setup. A plexiglass container is filled with mineral oil. A rotation stage and a focused transducer are immersed inside it in the same $x-y$ plane. The sample can be set in the rotation stage. The transducer is used to detect the thermoacoustic signal from the sample. The angle panel indicates the angle between the acoustic axis of the transducer and the rotation radius. We manually turn the transducer to point to one direction. A step motor directly drives the rotation stage while the transducer is fixed. Obviously, this is equivalent to a transducer that rotationally scans around the sample with a manual sector scan. The transducer (V314, Panametrics) has a central frequency of $1 \mathrm{MHz}$, a bandwidth of $0.6 \mathrm{MHz}$, a diameter of $1.9 \mathrm{~cm}$, a focal length of $2.5 \mathrm{~cm}$ at $1 \mathrm{MHz}$, a $3-\mathrm{dB}$ focal diameter of $2.1 \mathrm{~mm}$, and a focal zone of $1.76 \mathrm{~cm}$ along the acoustic axis.

The microwave pulses transmitted from a 3-GHz microwave generator have a pulse energy of $10 \mathrm{~mJ}$ and a pulse width of $0.5 \mu \mathrm{s}$. A function generator (Protek, B-180) is used to trigger the microwave generator, control its pulse repetition frequency, and synchronize the oscilloscope sampling. Microwave energy is delivered to the sample by a rectangular wave guide with a cross section of $72 \mathrm{~mm} \times 34 \mathrm{~mm}$.

A personal computer is used to control the step motor in rotating the sample. The signal from the transducer is first amplified through a pulse amplifier, then recorded and aver- 

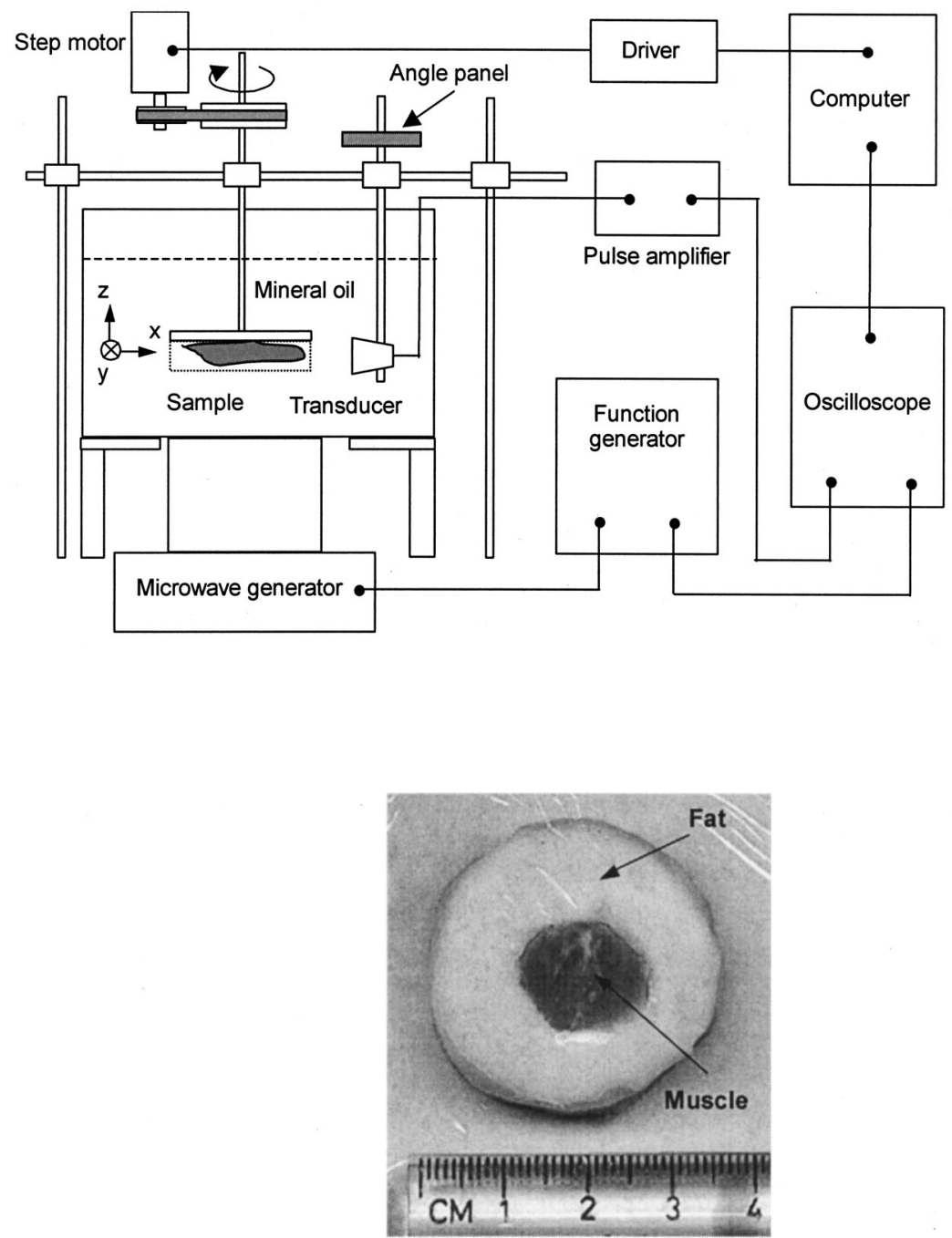

(a)
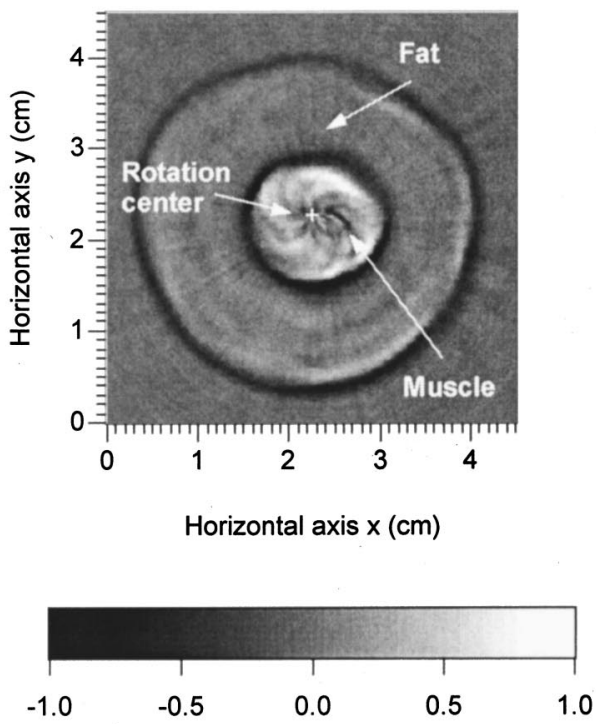

Piezoelectric signal (a.u.)

(b)
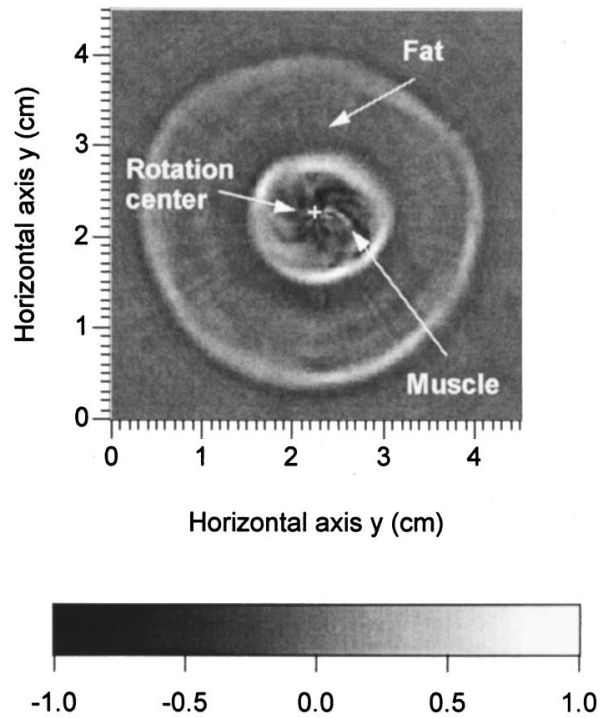

Derivative of piezoelectric signal (a.u.)

(c)
FIG. 3. Experimental setup.

FIG. 4. (a) Cross section of a sample on the $x-y$ plane; 2D constructed images of the $x-y$ cross section of the sample (b) from the piezoelectric signals and (c) from the first derivative of the piezoelectric signals. 


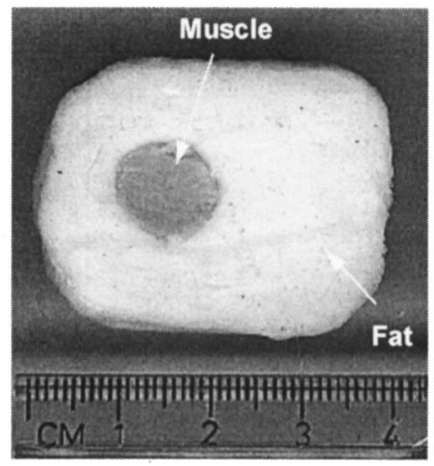

(a)
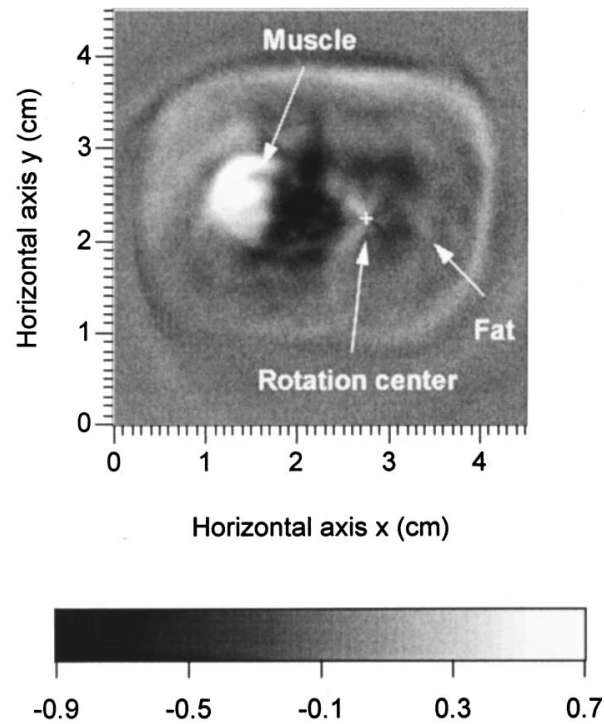

Piezoelectric signal (a.u.)

(b)
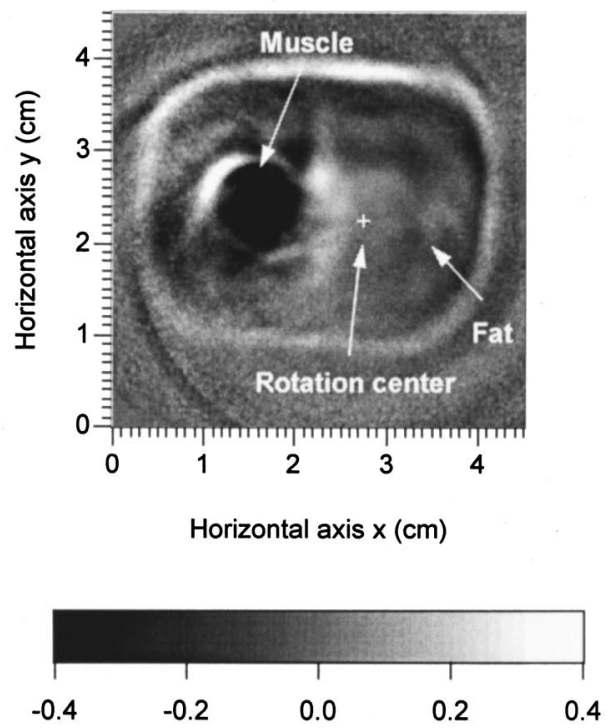

Derivative of piezoelectric signal (a.u.)
FIG. 5. (a) Cross section of a sample on the $x-y$ plane; 2D constructed images of the $x-y$ cross section of the sample (b) from the piezoelectric signals and (c) from the first derivative of the piezoelectric signals. aged 100 times by an oscilloscope (TDS640A, Tektronix), and finally transferred to a personal computer for imaging.

\section{RESULTS AND DISCUSSION}

The first sample we tested was a piece of muscle tissuecentered in a fat disc as shown in Fig. 4(a)-which was cut across and photographed after the experiment. Both the muscle and fat were cut from pork. The maximum diameter of the sample was $38 \mathrm{~mm}$, and the thickness was $5 \mathrm{~mm}$. The rotation center was set inside the muscle. The transducer rotationally scanned the sample (only in the rotation-radius directions) from 0 to 360 degrees with a step size of 2.25 degrees. We then used these 160 scan lines to calculate a 2D cross-sectional image with linear interpolations. Good agreement between the original profile in Fig. 4(a) and the constructed images in Figs. 4(b) and 4(c) was obtained, where Figs. 4(b) and 4(c) were computed from the measured piezoelectric signals and the first derivative of those, respectively. The boundaries between the fat and muscle or oil are clearly imaged. This is because when the rotation center is in the center of the sample, each scan line is nearly perpendicular to the boundaries of the muscle or fat, and the transducer can receive sufficient thermoacoustic signals from the radius directions alone for imaging.

The second sample had a structure as shown in Fig. 5(a), which was also cut across and photographed after the experiment. The fat was first cut from pork: the maximum diameter of the sample was $39 \mathrm{~mm}$; and the thickness was $5 \mathrm{~mm}$. Then we cut away a hole far away from its center and filled in a small piece of muscle, which was also cut from pork. The muscle size was about $10 \mathrm{~mm}$ in diameter and $5 \mathrm{~mm}$ in thickness. The rotation center was set outside the muscle. The rotation radius was $12.5 \mathrm{~cm}$, which included the length of the transducer. The transducer rotationally scanned the sample from 0 to 360 degrees with a step size of 2.25 degrees, and at each stop a set of sector directions from -12 to 12 degrees with a step size of 2 degrees was scanned. Totally, 2080 scan lines were acquired to construct 2D cross-section images. Figure 5(b) was constructed directly from the piezoelectric signals, which was in good agreement with the origi- 
nal profile in Fig. 5(a). The boundaries between the fat and muscle or oil are clearly visible, except the rear boundary of the muscle. Figure 5(c) was constructed from the first derivatives of the piezoelectric signals, which agreed with the original profile of Fig. 5(a) very well. The boundary of the muscle was clearer than that in Fig. 5(b). In particular, the sizes and locations of the muscle and fat zones agreed with the original shapes. It indicates that the first derivative produces a better mapping of the microwave absorption distribution than the piezoelectric signal, i.e., the thermoacoustic pressure. Due to the high microwave absorption in muscle and the low absorption in fat, the muscle and fat can be differentiated by their "brightness" in the images.

In the above examples, the size deviations between the original objects and their constructed images are about 2 $\mathrm{mm}$, which agree with the theoretical spatial resolution approximations as follows. The axial resolution along the acoustic axis is dependent on the width of the microwave pulse $(0.5 \mu \mathrm{s})$ plus the width of the impulse response of the transducer $(1.5 \mu \mathrm{s})$; therefore, it should be approximately 3 $\mathrm{mm}$, because the velocity of the acoustic waves in the tissue is about $1.5 \mathrm{~mm} / \mu \mathrm{s}$. The lateral resolution is determined by the focal diameter of the focused transducer, $\sim 2 \mathrm{~mm}$ for the $1 \mathrm{MHz}$ transducer used in the above experiments. The use of shorter microwave pulses and narrower focal diameter ultrasonic transducers can improve the spatial resolution. ${ }^{3}$

\section{CONCLUSION}

Microwave-induced thermoacoustic tomography of inhomogeneous tissues by using multiple sector scans was studied. Cross-sectional images can be obtained by a few straightforward calculations from the temporal data acquired by a focused transducer rotationally sector scanning the samples. The experiments show that the constructed images are in good agreement with the original cross-section profiles of the samples, and the boundaries between different tissues are clearly imaged. Results indicate that this technique is a powerful imaging method with good spatial resolution that can be used for the investigation of inhomogeneous tissues. In the future, a circular array could be used to replace the single transducer, and some numerical compensation methods could be introduced to improve the spatial resolution further.

\section{ACKNOWLEDGMENTS}

This project was sponsored in part by the U.S. Army Medical Research and Materiel Command Grant No. DAMD17-00-1-0455, the National Institutes of Health Grant No. R01 CA71980, the National Science Foundation Grant No. BES-9734491, and Texas Higher Education Coordinating Board Grant No. ARP 000512-0123-1999.

a) Author to whom all correspondence should be addressed. Electronic mail: lwang@tamu.edu; URL:http://oilab.tamu.edu

${ }^{1}$ R. A. Kruger, K. K. Kopecky, A. M. Aisen, D. R. Reinecke, G. A. Kruger, and W. L. Kiser, "Thermoacoustic CT with radio waves: A medical imaging paradigm,” Radiology 211, 275-278 (1999).

${ }^{2} \mathrm{G}$. $\mathrm{Ku}$ and L. V. Wang, "Scanning thermoacoustic tomography in biological tissues," Med. Phys. 27, 1195-1202 (2000).

${ }^{3}$ G. Ku and L. V. Wang, "Scanning microwave-induced thermoacoustic tomography: Signal, resolution, and contrast," Med. Phys. 28, 4-10 (2001).

${ }^{4}$ Medical Applications of Microwave Imaging, edited by L. E. Larsen and J. H. Jacobi (IEEE, Piscataway, NJ, 1986).

${ }^{5}$ P. M. Meaney, K. D. Paulsen, and J. T. Chang, "Near-field microwave imaging of biologically-based materials using a monopole transceiver system," IEEE Trans. Microwave Theory Tech. 46, 31-45 (1998).

${ }^{6}$ P. N. T. Wells, "Ultrasonic imagining of the human body," Rep. Prog. Phys. 62, 671-722 (1999).

${ }^{7}$ G. J. Diebold, T. Sun, and M. I. Khan, Photoacoustic waveforms generated by fluid bodies, in Photoacoustic and Photothermal Phenomena III, edited by D. Bicanic (Springer-Verlag, Berlin, Heidelberg, 1992), pp. 263-269. 